\title{
Histo-pathological correlation of BI-RADS 4 lesions on mammography with emphasis on microcalcification patterns
}

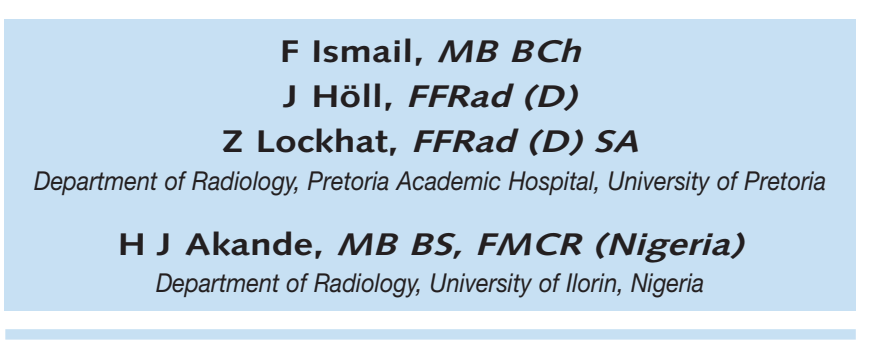

\section{Abstract}

A retrospective study of 20 patients with breast imaging reporting and data system (BI-RADS) 4 lesions, who underwent biopsy, was undertaken and a comparison with histological analyses was performed to compare the radiological appearance of benign and malignant microcalcification patterns with the final histology reports.

The results showed that although the study was not statistically significant owing to an insufficient study population, interesting trends developed which suggest that there are deficiencies in the evaluation of microcalcifications using the BI-RADS, as some lesions that were thought to have benign calcification patterns were actually malignant, and vice versa. It is hoped that further study in this field will highlight problematic areas in the evaluation of microcalcifications.

\section{Introduction}

The study was undertaken because many patients who had suspected radiological evidence of malignant calcification patterns had benign disease on histology, and vice versa. Similar problems in other parts of the world have been described in the literature.

In screening and diagnostic mammography, the presence of microcalcifications is an important marker for ductal carcinoma in situ (DCIS). In screening mammography, a palpable mass is not present and as many as $30-50 \%$ of nonpalpable breast cancers present themselves as clusters of microcalcifications alone. ${ }^{1}$ It is therefore important to develop good radiological and histopathological correlation.
Two classification systems in the evaluation of microcalcifications now exist, viz. Le Gal's classification and the BI-RADS, which was developed by the American College of Radiology (ACR) to standardise mammographic reporting.

Depending on the radiological appearance on mammography and ultrasound, patients may require further evaluation. The decision as to the type of biopsy needed and the follow-up interval can be controversial. The problem is compounded by the fact that microcalcification patterns may vary and do not always indicate breast cancer. There is a fine line between aggressive lesion biopsy, which affects patient morbidity, and the wait-and-watch approach during which time cancers may grow.

\section{Materials and methods}

A retrospective study of 20 patients with BI-RADS 4 lesions with microcalcifications on mammography, who underwent biopsies, was performed. Biopsies were done using a stereotactic technique with a Magnum true-cut device or a Vacora vacuum-assisted biopsy device.

A detailed history on every patient referred to our department is taken prior to any investigation, on a standardised form. Data were collected from this form as well as from mammogram, ultrasound and histology reports. All patients were classified according to the BI-RADS, and all patients had a combination of a mass (clinical or

\section{Table I. Histology of benign disease}

\begin{tabular}{lc|}
\multicolumn{2}{c}{ Table I. Histology of benign disease } \\
\hline & $\boldsymbol{N}$ \\
Epithelial hyperplasia & 1 \\
Chronic infection & 2 \\
No malignancy & 3 \\
Fibroadenosis & 2 \\
Intraductal papilloma & 1 \\
Radial scar & 1 \\
\end{tabular}

\begin{tabular}{ll|}
\hline & Table II. Clinical and other relevant history in patients with ductal carcinoma \\
\hline Age & $20-29=2,40-49=1,50-59=2,60-69=2,70-79=4$ \\
No children & 1 \\
Never breast-fed & 1 \\
Positive family history & 5 \\
Post-menopausal & 5 \\
Breast mass & 8 \\
Ductal carcinoma grades & DCIS: 7 (1 comedo-type DCIS), infiltrating DCIS: 4 \\
\end{tabular}


Table III. Comparison of calcification patterns with final histology results

\section{Mammographic description}

1. Cluster, pleomorphic and amorphic - calcification pattern malignant

2. Pleomorphic, well circumscribed - calcification pattern benign

3. Heterogeneous (with spiculated mass) - calcification pattern malignant

4. Scattered punctuate, linear and amorphous (with mass) - calcification pattern benign

5. Single round calcification (with mass) - calcification pattern benign

6. Clumped, dense calcification pattern (with mass) - calcification pattern benign

7. Clusters of heterogeneous, punctuate calcifications - calcification pattern benign

8. Amorphic calcifications in ill-defined lesion - calcification pattern malignant

9. Linear and punctuate calcifications in area of architectural distortion - calcification pattern malignant

10. Clusters of linear and punctuate calcifications (with spiculated mass) - calcification pattern malignant

11. Dense, clumped pleomorphic calcifications (with mass) - calcification pattern malignant

12. Rod-shaped, scattered calcifications (with skin retraction) - calcification pattern benign

13A. Clustered, pleomorphic punctuate calcifications (with mass) - calcification pattern malignant

13B. Large dystrophic calcifications, punctate calcifications dispersed (with mass) - calcification pattern benign

13C. Dense clustered calcifications (with mass) - calcification pattern benign

14. Segmental, pleomorphic calcifications - calcification pattern malignant

15. Scattered calcifications (with architectural distortion) - calcification pattern benign

16. Diffuse punctate calcifications (with mass) - calcification pattern malignant

17. Segmental clustered pleomorphic and punctate calcifications (no mass) - calcification pattern malignant

18. Scattered punctate calcifications (with mass) - calcification pattern benign

19. Dense clusters of amorphic calcifications (with mass) - calcification pattern malignant

20. Few clumped amorphic calcifications (architectural distortion, no masses in I and II) - calcification pattern malignant

\section{Histological result}

Fibrosis

Chronic infection

DCIS

DCIS

DCIS

Acute-on-chronic infection

Infiltrating DC

Ductal ectasia

No malignancy

No malignancy

DCIS

Infiltrating DC

Fibroadenosis
$13 \mathrm{~A}$ - Invasive DC
$13 \mathrm{~B}-\mathrm{DCIS}$
$13 \mathrm{C}$ - Invasive DC

DCIS - comedo type

Fibroadenosis

Invasive DC

Invasive ductal carcinoma - comedo type

DCIS

Radial scar with focal MC

$\mathrm{I}=$ No abnormality detected

II = No abnormalities detected 
on mammography) architectural distortion and microcalcifications. Emphasis was placed on the pattern of microcalcifications present.

\section{Results}

Of the 20 patients who underwent biopsies, a total of 23 lesions were biopsied, with 2 patients having multifocal disease (10\%). The age distribution of the patients is shown in Fig. 8. Of the 23 lesions biopsied, 13 demonstrated ductal carcinoma (56.5\%), which was found in 11 of the 20 patients.

The histological results of the 10 lesions that were negative for malignancy are shown in Table I. Table II sets out other relevant history of the 11 patients with ductal carcinoma. Of the 11 patients who presented with a clinical breast mass, 8 were malignant $(72 \%)$ and 3 were benign (28\%). Of the 9 patients who did not present with a clinical mass, 6 presented with a spiculated mass on mammography, of which 4 (66.6\%) were malignant and 2 (33.3\%) demonstrated fibroadenosis with calcifications.

The calcification patterns, independently of other features of benign or malignant disease and comparison with histological results, were analysed (Table III). Of a total of 23 lesions, 13 had a suspected malignant calcification pattern, and $10 \mathrm{had}$ a suspected benign calcification pattern. Of the 13 with a suspected malignant calcification pattern, $6(46 \%)$ were benign on histological analysis. Of these, 1 had an associated mass, 2 had associated areas of architectural distortion, and 1 had an associated ill-defined region of asymmetry. Of the 10 with a suspected benign calcification pattern, $6(60 \%)$ were malignant on histological analysis, with a mass present in 5 (83\%) of these 6 patients.

The odds ratio was 1.5 with a $95 \%$ confidence interval (CI) 0.19 11.94; not significant. The relative risk was 1.2 with $95 \%$ CI 0.54 - 2.68; not significant. The $p$ value was 0.65 (Mantel-Haenszel); not statistically significant.

Analysis of patients presenting with a mass (with microcalcifications) on mammography and comparing this with histological results was undertaken. Of the 23 lesions, 13 presented with a mammographic (not necessarily clinical) mass and 10 without a mass. Of the 13 patients with a mass, malignancy was found in 10 patients (76.9\%); of the 10 lesions without a mass, malignancy was found in 3 lesions (30\%).

\section{Analysis of results}

The study sample was too small to offer statistical significance, therefore continuation of the study is essential. Interesting early trends were noted from this study, as follows:

- Regarding age, the highest incidence of microcalcifications occurred in patients older than 50, with a second peak in the 20 - 29-year-old age group; further study as to the possible aetiology in this age group is required.

- In patients with a clinical breast mass, there was a higher risk of malignancy (72\%).

- In patients where a mass was found on mammography (with microcalcifications), there is a higher risk of malignancy (66.6\%).

- In assessing calcification patterns using the BI-RADS, some lesions that were thought to have benign calcification patterns were actually malignant (60\%), and vice versa $(46 \%)$. Further study in this field is required as the study sample is too small to have statistical significance.

- In patients with segmental distribution of microcalcifications, there is an increased prevalence of comedo-type DCIS. Although this is described in older patients, both our patients were in the 20 - 29-yearold age group.

\section{Discussion}

The BI-RADS was developed in 1985 to standardise mammographic reporting and follow-up. ${ }^{2}$ On systematic review of the presenting mammogram, the radiologist is presented with many patterns such as a mass, asymmetry (focal or globular), calcifications, architectural distortion, skin, sub-areola and pectoral muscle contour distortion, as well as lymphadenopathy. Each abnormality is assessed systematically together with the patient's history and ultrasound findings to come to one of 6 final conclusions (BI-RADS is divided into 6 categories): ${ }^{2}$

0 : Recall for further analysis or request for previous studies

1: Negative

2: Benign finding - routine follow-up recommended

3: Probably benign finding - short follow-up recommended

4a: Low suspicion for malignancy

$4 \mathrm{~b}$ : Intermediate suspicion for malignancy

4c: Moderate concern but not classic for malignancy. Biopsy is considered for stage 4 lesions.

5: High suspicion for malignancy

6: Biopsy-proven malignancy prior to definitive therapy.

Orel and colleagues found positive predictive values for malignancy in the categories as follows:
2: $0 \%$
3: $2 \%$
4: $30 \%$
5: $97 \%$.

In terms of calcifications, there are 3 main categories to consider: pleomorphic, linear and linear-branching types have the highest probability for malignancy. Pleomorphic calcifications are $<0.5 \mathrm{~mm}$ in size and have different shapes. ${ }^{2}$ Amorphous calcifications have an illdefined edge and may be problematic to classify. These calcifications have an intermediate probability for malignancy. ${ }^{2}$ Coarse heterogeneous calcifications are irregular in shape, conspicuous and $>0.5 \mathrm{~mm}$ in size; this type is the least suspicious for malignancy. ${ }^{2}$

In our study, the evaluation of microcalcifications was problematic, with $60 \%$ of calcifications thought to be benign having been proven on mammographic assessment to be malignant on biopsy, and $46 \%$ of malignant calcification patterns proven to be benign on biopsy. This has significant implications for patient management. Further clarity is required in this field to improve categorisation of microcalcifications in the BI-RADS.

Segmental calcification patterns do not have a separate category in BI-RADS; this may need consideration in the future as there is an increased risk of malignancy, especially the comedo type, as demonstrated by our study.

Le Gal's classification is concerned with the morphology of calcification. There are 5 types: 


$\begin{array}{ll}\text { 1. Annular } & \\ \text { 2. Regularly punctiform } & \vdots \\ \text { 4. Dusty } & \end{array}$

A study by Fondrinier et al. found the incidence of malignancy according to Le Gal's classification to be as follows: ${ }^{4}$

1. Annular - 0\%

2. Regularly punctiform $-27 \%$

3. Dusty $-32 \%$

4. Irregularly punctiform $-65 \%$

5. Vermicular $-100 \%$.

A study by Gulsun et al. compared the positive predictive value (PPV) of Le Gal's classification and the BI-RADS classification in terms of identification of malignancy, and concluded that both did not succeed in reducing the ambiguity in assessment of breast calcifications but that they are useful for standardisation of reports. ${ }^{1}$

Another study by Yunus et al. used the Le Gal classification to show that the statistically significant criteria for malignant calcifications were irregularity of size and density, linear or branched shape, or Le Gal Type $\mathrm{V}$ distribution; also, at least 30 microcalcifications per cluster or $10 / \mathrm{cm}$ were found to be important criteria for malignancy. ${ }^{5}$

Atypical ductal hyperplasia (ADH), lobular carcinoma in situ (LCIS) and atypical lobular hyperplasia (ALH) may also present with calcifications that may exhibit high density, clustered punctuate calcification and tending to lack the characteristic features of DCIS such as rod shapes, ductal distribution and branching. ${ }^{6}$

DCIS can also present as a stellate mass without calcifications. It may occur with DCIS alone or in association with a complex sclerosing lesion (CSL). Reiff et al. performed a retrospective study on 677 women with DCIS and found that $8 \%$ had a stellate lesion without microcalcifications. ${ }^{6}$

All patients in our study had microcalcifications, and those with clinical or mammographic masses had a higher risk of malignancy of $72 \%$ and $66.6 \%$ respectively. The presence of a mass pushed the BIRADS classification to group 4, which required biopsy, even though the calcification pattern was benign. This enabled biopsy of benign calcification patterns and correlation with histology, and $46 \%$ of the masses were found to be malignant.

The ultrasound approach to calcifications includes identification of BI-RADS 2 calcifications (benign) such as oil cysts and milk of calcium with other calcifications requiring mammographic evaluation. Therefore, the current role of ultrasound is to exclude benign calcifications only.

On ultrasound, the features of DCIS are as follows: ${ }^{7}$

1. Hypoechoic mass with or without calcifications

2. Dilated ducts with or without calcifications

3. Intraductal mass/hypoechoic material with or without calcifications

4. Ducts with irregularly thickened walls

5. Calcifications without a mass
6. Shadowing, which may or may not be associated with the mass or calcification.

Hashimoto ${ }^{7}$ also noted that the presence of posterior acoustic shadowing of a calcification is variable and its presence is highly dependent on transducer frequency (more common when using probes of higher frequency, i.e. $13 \mathrm{MHz}$ or more).

Magnetic resonance imaging (MRI) is not helpful in differentiating between benign and malignant calcifications, and MRI has specific indications in breast cancer such as the detection of multicentric, multifocal or contralateral breast cancer, or the detection of residual disease. Of note is that MRI is suggested in patients with occult cancer, i.e. patients who present with a positive lymph node biopsy but negative mammogram findings. ${ }^{8}$

With DCIS, MR spectroscopy may be beneficial when used with MRI. In one study, choline peaks were identified in a variety of cancer histologies including 16 invasive cancers and one DCIS. ${ }^{8,9}$

Raman spectroscopy is another method that is being tested to separate benign and malignant calcifications but is only used in vitro currently. The Type II microcalcifications consist primarily of calcium hydroxyapatite; it is this type of calcium that is associated with malignancy. Haka et al. have shown that the Type II microcalcifications manufactured in malignant ducts have a higher concentration of protein compared with calcium, and used this strategy to distinguish benign from malignant microcalcifications, using Raman spectroscopy. ${ }^{10}$

Other authors have developed mathematical algorithms to enable computer-generated detection of benign and malignant calcifications, and have concluded that the programme gives satisfying results and seems to be a promising tool for clinical use. ${ }^{11}$

\section{Conclusion}

The study population was insufficient to establish statistical significance but demonstrates interesting trends of some deficiencies in the evaluation of microcalcifications using BI-RADS. We plan to continue this study to improve mammographic sensitivity in the evaluation of microcalcifications.

We gratefully acknowledge the assistance of Mrs D Povey, Dr P Ligege, Dr A Janse van Rensburg and Dr S Khan.

1. Gulsun M, Demirkazik FB, Ariyurek M. Evaluation of breast microcalcifications according to breas imaging reporting and data system criteria and Le Gal's classification. Eur J Radiol 2003; 47: 227-231.

2. D'Orsi CJ, Newell MS. BI-RADS decoded. Detailed guidance on potentially confusing issues. Radiol Clin North Am 2007; 45: 751-763.

3. Orel SG, Kay N, Reynolds C, et al. BI-RADS categorization as a predictor of malignancy. Radiology 1999: 211: 845-850.

4. Fondrinier E, Lorimer G, Guerin-Boblet V, Bertrand A-F, Mayras C, Dauver N. Breast microcalcifications; multivariate analysis of radiological and clinical factors for carcinoma. World J Surg 2002; 26: 290-296.

5. Yunus M, Ahmed N, Masroor I, Yaqoob J. Mammographic criteria for the diagnostic value of microcalcifications in the detection of early breast cancer. J Pak Med Assoc 2004; 54(1): 24-29.

6. Reiff DB, Cooke J, Griffin M, Given-Wilson R. Ductal carcinoma in situ presenting as a stellate lesion on mammography. Clin Radiol 1994; 49: 396-399.

7. Hashimoto BE. Sonographic assessment of breast calcifications. Curr Probl Diagn Radiol 2006; 35: 213218.

8. Bartella L, Smith CS, Dershaw D, Liberman L. Imaging breast cancer. Radiol Clin North Am 2007; 45 : 45-67.

9. Bartella L, Morris EA, Dershaw D, et al. Proton MR spectroscopy with choline peak as a malignancy marker improves positive predictive value for breast cancer diagnosis: A preliminary study. Radiology 2006; 239: 686-692

10. Haka AS, Karen E, Shafer-Peltier E, et al. Identifying microcalcifications in benign and malignant breast lesions by probing differences in their chemical composition using Raman spectroscopy. Cancer Res 2002; 62: 5375-5380.

11. Strauss A, Sebbar A, Desarnaud S, Mouillard P. Automatic detection and segmentation of microcalcifications on digitized mammograms. Eng Medicine Biol Soc 1992; 5: 1938-1939. 


\section{ORIGINAL ARTICLE}

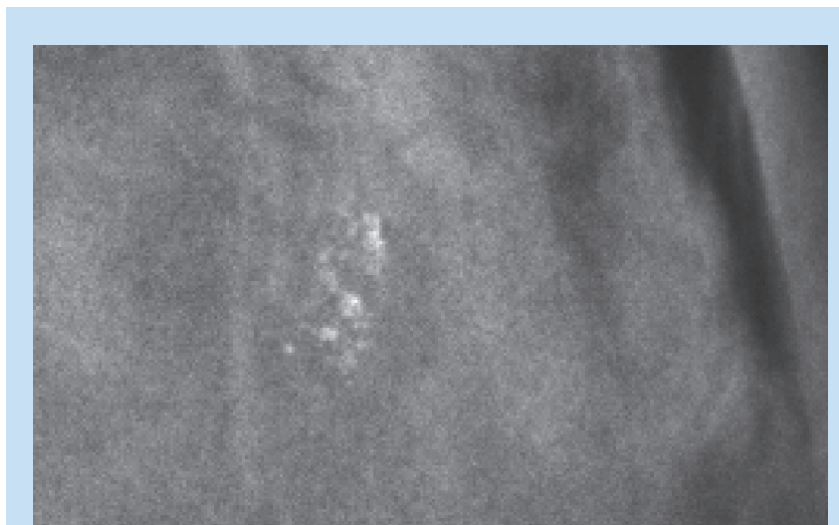

Fig. 1. Patient 1 - cluster of pleomorphic and amorphic calcifications; calcification pattern is malignant.

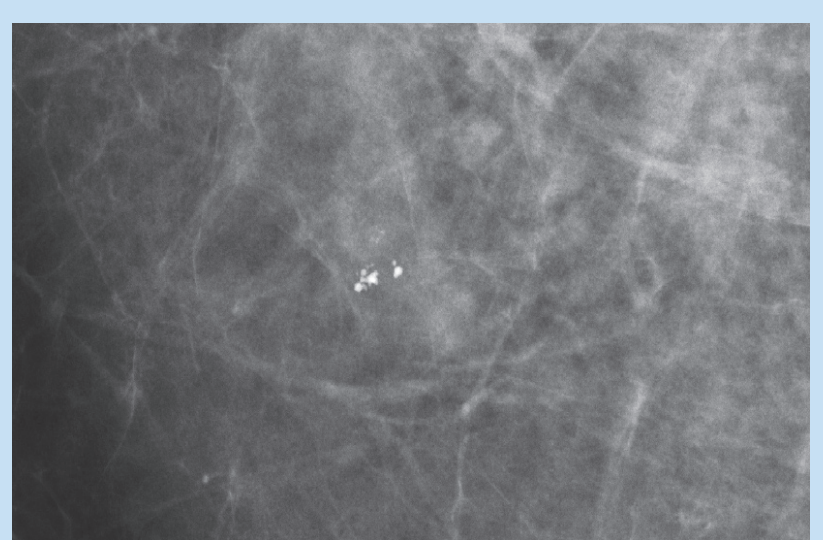

Fig. 2. Patient 2 - pleomorphic calcifications, well circumscribed; calcification pattern is benign.

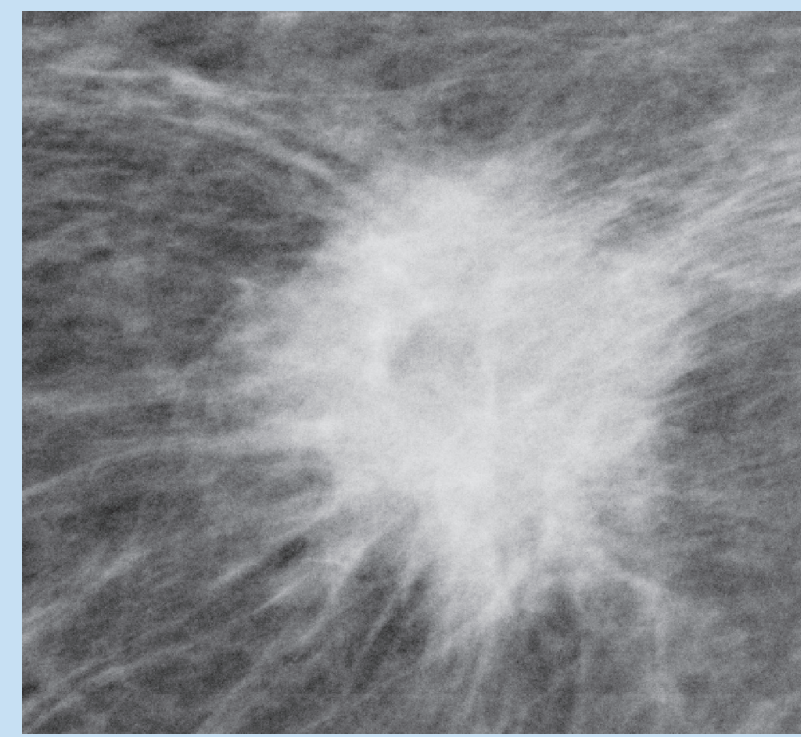

Fig. 3. Patient 3 - heterogeneous calcifications (with spiculated mass); calcification pattern is malignant.

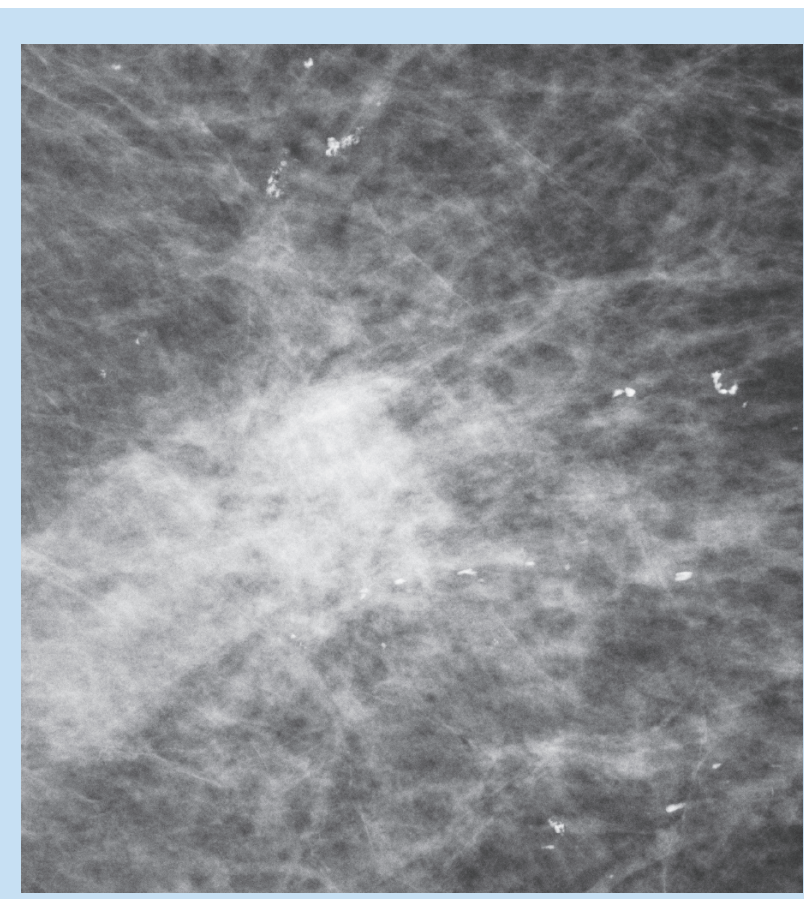

Fig. 4. Patient 4-scattered punctuate, linear and amorphous calcifications (with mass); calcification pattern is benign.

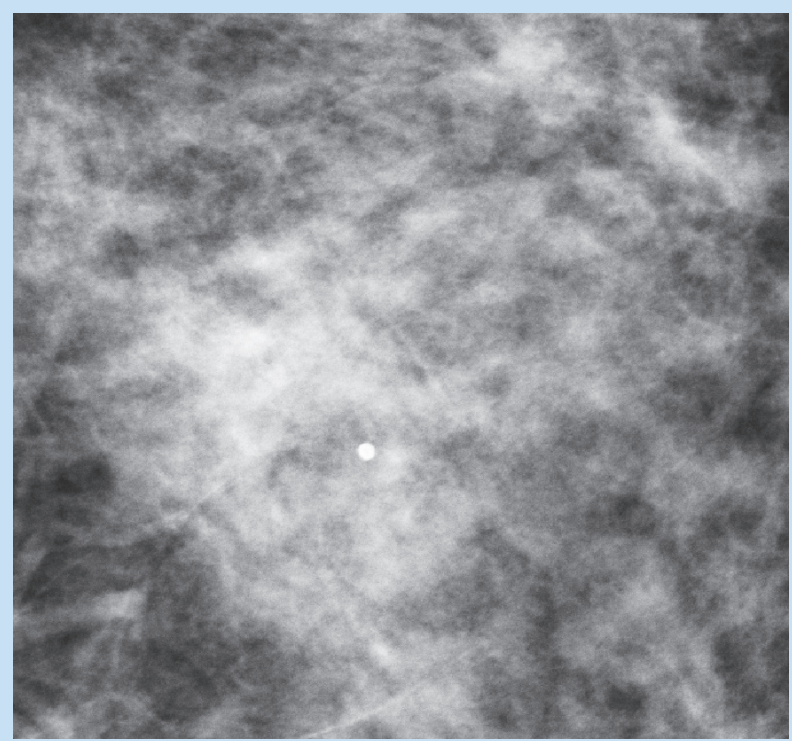

Fig. 5. Patient 5 - single round calcification (with mass); calcification pattern is benign. 


\section{ORIGINAL ARTICLE}

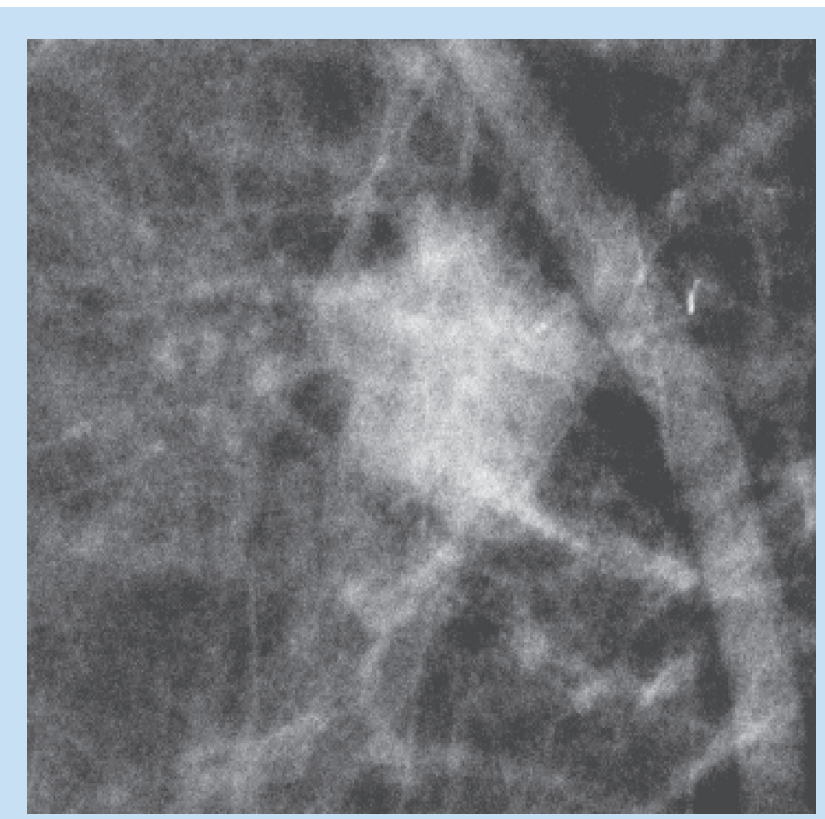

Fig. 6. Patient 6 - clumped, dense calcification pattern (with mass); calcification pattern is benign.

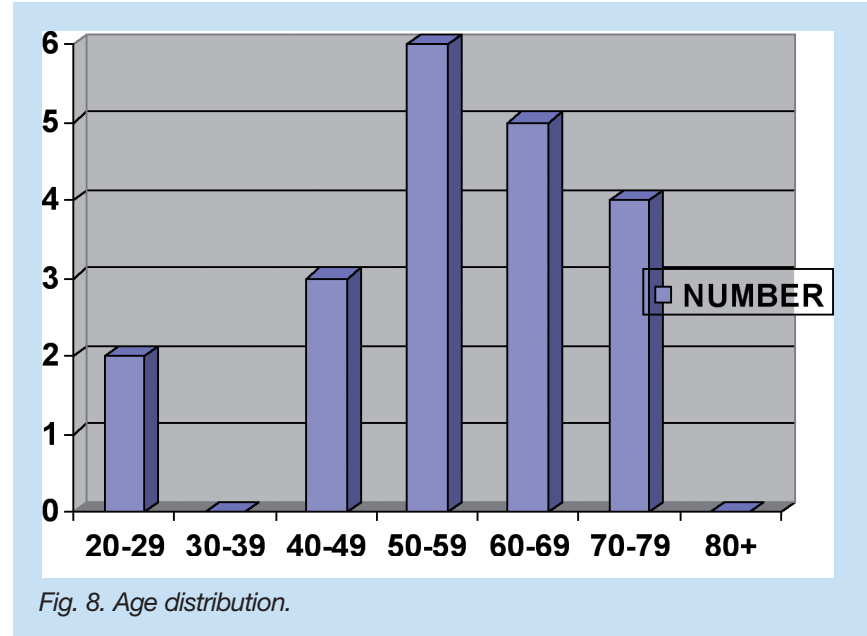

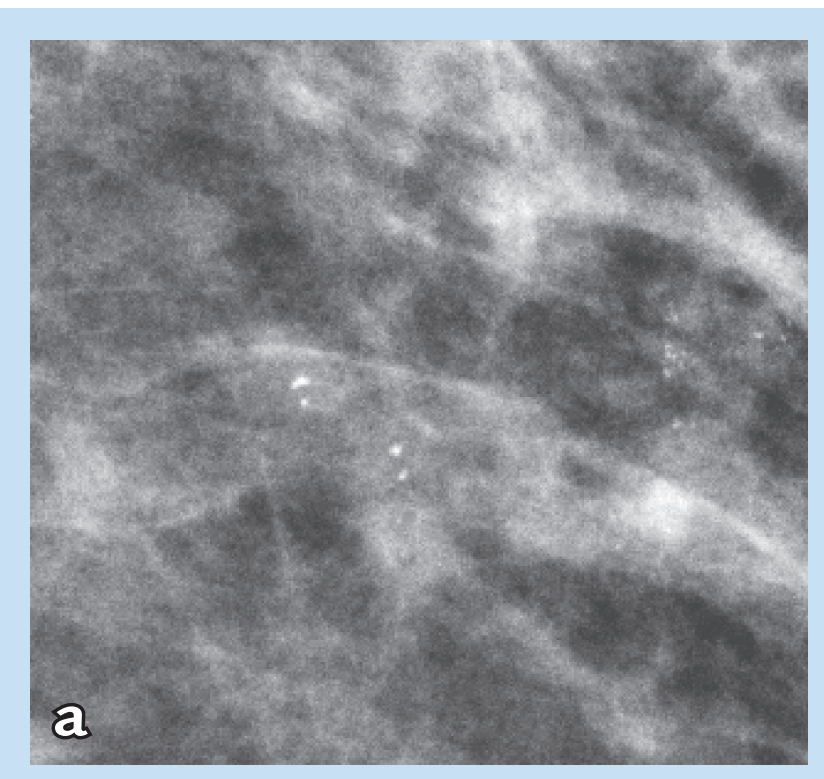

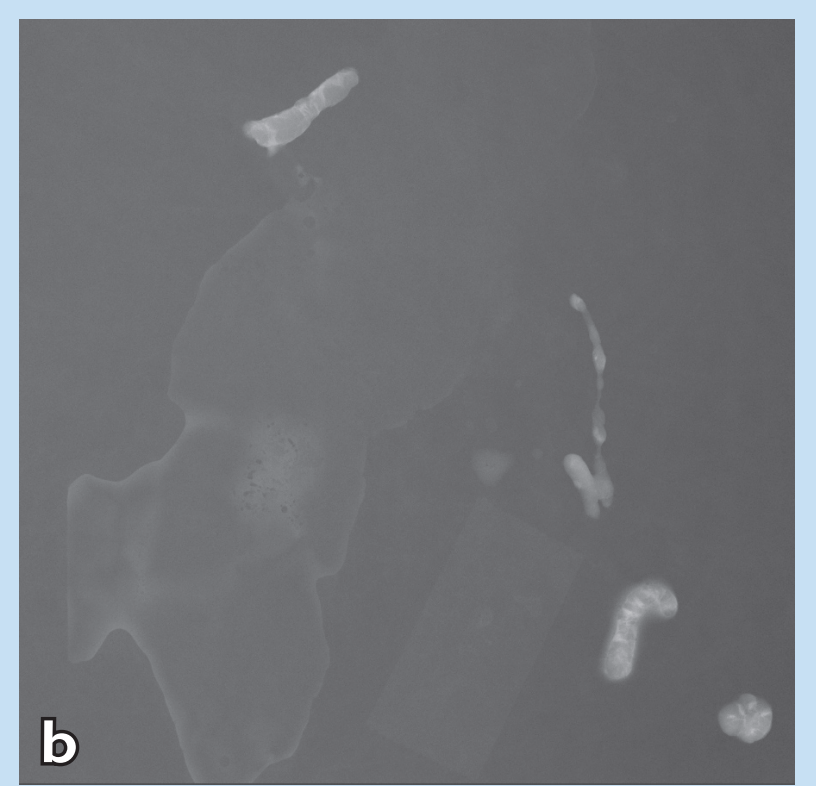

Fig. 7. Patient 7-mammogram (7a) showing clusters of heterogeneous, punctuate calcifications; calcification pattern is benign. A radiograph (7b) of specimens demonstrates that calcifications have been removed in biopsy. 